\title{
Progress in the treatment of NK-cell lymphoma/leukemia
}

\author{
Ayumi Fujimoto, Ritsuro Suzuki \\ Department of Hematology, Shimane University Hospital, Izumo, Shimane 693-8501, Japan.
}

Correspondence to: Dr. Ritsuro Suzuki, Department of Hematology, Shimane University Hospital, 89-1 En-ya cho, Izumo, Shimane 693-8501, Japan. E-mail: rsuzuki@med.shimane-u.ac.jp

How to cite this article: Fujimoto A, Suzuki R. Progress in the treatment of NK-cell lymphoma/leukemia. J Cancer Metastasis Treat 2021;7:66. https://dx.doi.org/10.20517/2394-4722.2021.157

Received: 4 Aug 2021 First Decision: 14 Sep 2021 Revised: 29 Sep 2021 Accepted: 29 Oct 2021 Published: 5 Dec 2021

Academic Editors: Lucio Miele, Lorenzo Leoncini, Ivory Ma Copy Editor: Xi-Jun Chen Production Editor: Xi-Jun Chen

\begin{abstract}
Natural killer (NK)/T cell lymphoma includes two major subtypes of disease, specifically extranodal NK/T cell lymphoma, nasal type (ENKL) and aggressive NK cell leukemia (ANKL). Both are strongly associated with EpsteinBarr virus and are prevalent in East Asia and Latin America. Except for that of limited-stage ENKL, the prognosis of both diseases was poor in the previous decade. The advent of non-anthracycline-based chemoradiotherapy has contributed to an improvement in ENKL prognosis, but there is still room for further treatment progress. Recently, the high efficacy of PD-1 antibody was reported in relapsed or refractory ENKL patients. This was later supported by the finding that PD-L1/PD-L2 genetic alterations are frequently observed in ENKL and ANKL patients. Due to the rarity of the disease, a standard treatment for ANKL remains to be established. Currently, allogeneic stem cell transplantation is the only curative treatment, and this is even applicable to chemo-resistant ANKL patients. In this review, we focus on recent treatment approaches for NK/T cell lymphomas including novel agents.
\end{abstract}

Keywords: Extranodal NK/T-cell lymphoma, nasal type, aggressive NK-cell leukemia, Epstein-Barr virus, hematopoietic stem cell transplantation, L-asparaginase

\section{INTRODUCTION}

Natural killer (NK) cell neoplasms are rare hematological malignancies, with two major subtypes of extranodal NK/T cell lymphoma, nasal type (ENKL) and aggressive NK-cell leukemia (ANKL) ${ }^{[1]}$. Both 
ENKL and ANKL are strongly associated with Epstein-Barr virus (EBV) ${ }^{[1]}$. The geographic distribution of ENKL is distinctive, with a particularly high prevalence in East Asia and South America. ENKL corresponds to approximately $2 \%-11 \%$ of cases among all lymphoma subtypes in East Asian countries, whereas the prevalence is less than $1 \%$ in the United States and Europe ${ }^{[2-4]}$. Although the distribution and incidence of ANKL are unclear due to its rarity, the prevalence is approximately one-sixth that of ENKL, accounting for fewer than $1 \%$ of cases among all malignant lymphomas ${ }^{[5]}$. ENKL is distinct from other lymphomas based on its unique characteristics, comprising the dominant involvement of the nasal cavity and nasopharynx. The majority of ENKL cases are limited to a location around the nose or upper aerodigestive tract, presenting with typical symptoms of nasal obstruction, epistaxis, or rhinorrhea. In contrast, ANKL is prevalent in the peripheral blood, bone marrow, liver, and spleen. Most patients suffer general symptoms, such as fever, general malaise, and loss of appetite. Clinical courses of advanced-stage ENKL and ANKL are very aggressive, often leading to disseminated intravascular coagulation and multiorgan dysfunction, thus resulting in a poor prognosis over the past decade. Recently, with an increasing understanding of the molecular pathogenesis of NK cell neoplasms, novel treatment strategies including targeted agents are expected to improve survival. In this review, we highlight recent clinical findings for ENKL and ANKL and discuss future prospects.

\section{CLINICAL CHARACTERISTICS OF NK/T CELL LYMPHOMA/LEUKEMIA}

The annual incidence of ENKL varies by ethnicity and geographically from 0.06 in the United States to 0.25 in Hong Kong per 100,000 population ${ }^{[3]}$. ENKL predominantly occurs in middle-aged individuals with a median age of 52-58 years ${ }^{[5-8]}$. The incidence is 1.5 times higher in males. Because ENKL commonly develops in extranodal sites and approximately $10 \%$ of ENKL cases are derived from $\gamma \delta$ or cytotoxic T cells $^{[\rho]}$, the disease is termed extranodal NK/T cell lymphoma in the World Health Organization (WHO) classification. The nasal or paranasal cavities and the upper aerodigestive tract are involved in more than $80 \%$ of ENKL cases, with approximately $70 \%$ of these being in a limited stage at diagnosis ${ }^{[5]}$. The patient's initial complaints are generally focal symptoms such as nasal obstruction, epistaxis, and rhinorrhea. In contrast, the remaining cases develop in extra-nasal sites such as the skin, gastrointestinal tract, lung, or other uncommon organs $s^{[5,7]}$. Approximately $60 \%$ of cases with an extra-nasal origin are at an advanced stage at diagnosis. Because ENKL develops predominantly in extranodal sites, stage III cases are rare, occurring in fewer than $5 \%$ of all cases. Therefore, most cases in advanced stage are diagnosed as stage IV. These cases follow an aggressive clinical course with hemophagocytic syndrome and/or disseminated intravascular coagulation.

ANKL is a rare leukemic form of NK cell neoplasms, which has been reported mainly in East Asian countries such as Japan, China, or South Korea. The incidence is 2-3 times higher in males. ANKL progresses very rapidly, and its very aggressive clinical course is reflected by its name, aggressive NK-cell leukemia $^{[10]}$. The median overall survival (OS) of ANKL is only a few months after diagnosis, which is the worst prognosis among all lymphoma subtype ${ }^{[11]}$. Interestingly, however, a subset of ANKL shows a subacute clinical course. These subacute-type patients demonstrate female predominance and better prognosis with a median OS of more than six months. The median age for ANKL development is 40-42 years old, which is more than 10 years younger than that of ENKL, but it is also distributed in an elderly population, older than 70 years of age ${ }^{[5,12]}$. Although most ANKL cases develop de novo, there are some cases that originate from EBV-associated T/NK lymphoproliferative disease, including chronic active EBV infection, which generally develops in a younger population ${ }^{[13]}$. Unlike ENKL, the clinical symptoms of ANKL are non-specific, including fever, general malaise, and loss of appetite. Most patients have hepatomegaly and splenomegaly; thus, increased levels of lactate dehydrogenase and liver enzymes are commonly observed. Therefore, the accurate diagnosis of ANKL is often difficult, and a postmortem 
diagnosis is sometimes made after an inaccurate diagnosis of acute hepatitis. Complete blood counts show pancytopenia, and the morphology of leukemic cells varies widely, from normal large granular lymphocyte to lymphoid cells with atypical nuclei and a prominent nucleolus. Occasionally, the percentage of tumor cells in the bone marrow and peripheral blood is much lower than that in acute myeloid or lymphoblastic leukemia, with median percentages of $22 \%$ and $8 \%$, respectively ${ }^{[12]}$. Therefore, it is important to consider ANKL as a differential diagnosis particularly in cases with fever of unknown origin, hepatosplenomegaly, or liver dysfunction.

The detection of EBV is required to make a diagnosis of ENKL based on the current WHO classification ${ }^{[1]}$, whereas this is not mandated for ANKL since approximately 10\%-15\% of ANKL cases are negative for $\mathrm{EBV}^{[12,14]}$. The differences in clinical course and prognosis between ANKL patients with and without EBV infection are also controversial ${ }^{[12,14,15]}$.

\section{PROGNOSIS AND PROGNOSTIC MODELS}

Several recent reports show that the prognosis of limited-stage ENKL patients has been improved through the introduction of several effective treatments in the past decade. However, the prognosis of advancedstage ENKL patients is still unsatisfactory ${ }^{[7,8,16]}$. The patients with limited-stage ENKL have a significantly better prognosis than those with advanced-stage $\mathrm{ENKL}^{[7]}$. In the largest retrospective study of $358 \mathrm{ENKL}$ patients, the five-year OS and progression-free survival (PFS) of limited-stage ENKL patients were $68 \%$ and $56 \%$, respectively. In addition, the prognosis was significantly better for the limited-stage ENKL patients treated between 2010 and 2013 than for those treated between 2000 and 2009 (five-year OS of 79\% and 63\%, respectively $)^{[7]}$. One of the reasons for this survival improvement is that the rate of limited-stage patients treated with effective chemoradiotherapies has increased ${ }^{[16]}$. More recently, the prognosis of limited-stage ENKL, when achieving event-free survival at 24 months after diagnosis, was demonstrated to be almost equivalent to that of the age- and sex-matched general population ${ }^{[17]}$. In contrast, the prognosis of advancedstage ENKL is still poor with five-year OS and PFS values of $24 \%$ and $16 \%$, respectively ${ }^{[7]}$. Although the introduction of L-asparaginase ( $\mathrm{L}$-asp)-containing chemotherapy has improved the OS, significant improvements in clinical practice have not been obtained yet ${ }^{[16]}$.

The most popular prognostic model for ENKL is the prognostic index of NK lymphoma (PINK) ${ }^{[18]}$. This model was developed to predict the prognosis for treatment-naïve ENKL patients who received nonanthracycline-based chemotherapies with curative intent. Based on four prognostic factors, including age older than 60 years, stage III or IV, distant lymph-node involvement, and non-nasal type disease, patients are stratified into low risk (0), intermediate risk (1), and high risk (2-4) categories, with estimated three-year OS of $81 \%, 62 \%$, and $25 \%$, respectively. PINK-E, a prognostic model including EBV-DNA data on PINK, also links risk factors and OS. The international prognostic index and NK/T cell lymphoma prognostic index, previously developed prognostic models, were based on patient data of the anthracycline era, and these do not provide an accurate prognosis for patients receiving recent non-anthracycline-based chemotherapies $^{[7,19]}$.

The prognosis of ENKL differs by the site of origin, specifically nasal or extranasal. The prognosis of patients with an extranasal origin is significantly worse than that of patients with a nasal origin based on several studies ${ }^{[6,8]}$. The largest prospective cohort study of ENKL patients, from the International $\mathrm{T}$ cell Project, showed an OS at five years of $54 \%$ in patients with nasal origin and $34 \%$ in those with extranasal origin ( $\log$-rank $P=0.019)^{[8]}$. However, the impact of the site of disease origin, nasal or extranasal, on OS is controversial. Because the percentage of advanced stage disease is generally higher in patients with extranasal origin than in those with nasal origin, the disease stage could be a confounding factor when 
evaluating the prognosis by the site of disease origin. In fact, another study reported that prognosis does not significantly differ between the two groups in subgroup analyses by disease stage ${ }^{[20]}$.

There are no prognostic models for ANKL. In general, the prognosis of this disease is extremely poor, with a median OS of 2-3 months ${ }^{[5,1,1,2]}$, except for that of the subacute-type ANKL ${ }^{[21]}$. Allogeneic stem cell transplantation is the only curative treatment and could be the most important factor for survival. Patients with subacute ANKL have symptoms of infectious mononucleosis for more than 90 days prior to the fulminant onset, and the prognosis is significantly better than that of typical ANKL, with a median OS of 214 days $^{[21]}$. ANKL originating from EBV-associated NK/T cell lymphoproliferative diseases such as chronic active EBV infection has been reported ${ }^{[13]}$, but its clinical course and prognosis are not well known.

\section{TREATMENT OF NK-CELL LYMPHOMA}

The recommended algorithm of treatments for ENKL patients is shown in Figure 1. The scheme is divided based on the extent of disease (limited $v s$. advanced). The history of treatment development is further shown in Table 1.

\section{Limited-stage ENKL}

For limited-stage ENKL, concurrent chemoradiotherapy (CCRT) is recommended as a first-line therapy. Radiotherapy (RT) has been used as an effective treatment for limited-stage ENKL since the $1980 \mathrm{~s}^{[22]}$. Although RT-containing treatment provides significantly better outcomes for limited-stage ENKL ${ }^{[23]}$, RT alone frequently induces local or systemic treatment failure in more than $50 \%$ of patient ${ }^{[24-26]}$. Several studies have shown that the recommended dose of RT that is necessary for local control of limited-stage ENKL is 50 Gy or more ${ }^{[23,27,28]}$. A combination of CHOP (cyclophosphamide, doxorubicin, vincristine, and prednisone) and RT is an established treatment for localized non-Hodgkin lymphoma, and it is also used to treat ENKL patients ${ }^{[29]}$. However, because normal and abnormal NK cells express multidrug resistant (MDR)-associated P-glycoprotein ${ }^{[30,31]}$, anthracycline-containing regimens such as CHOP are not satisfactory for treating ENKL patients, with a five-year OS of less than $50 \%$, even combined with $\mathrm{RT}^{[6,24,32,33]}$. In addition, chemotherapy followed by RT was shown to yield a worse response rate than RT followed by chemotherapy for limited-stage ENKL in a few studies ${ }^{[2,3,34,35]}$. Based on these experiences, several CCRT regimens have been developed as novel anti-MDR treatments for limited-stage ENKL patients since the early $2000 \mathrm{~s}^{[36-38]}$. These regimens consist of non-MDR-associated anti-cancer agents, such as platinum derivatives, and ifosfamide (IFM), L-asp which has in vitro sensitivity against an NK cell tumor cell line ${ }^{[39]}$, or etoposide (ETP) which is known to be effective against EBV-associated hemophagocytic syndrome ${ }^{[40,41]}$. One of the most popular CCRT regimens is RT-2/3DeVIC (dexamethasone, ETP, IFM, and carboplatin) ${ }^{[36]}$, which includes concurrent RT of 50-50.4 Gy in 25-28 fractions with three cycles of a two-third dose of DeVIC. The efficacy of RT-2/3DeVIC therapy for newly diagnosed stage IE or contiguous IIE ENKL patients is better with a two-year OS of $78 \%$ and an overall response rate (ORR) of $81 \%$, relative to the historical data. The adverse events (AEs) of RT-2/3DeVIC are acceptable. There have been no treatmentrelated deaths and only neutropenia was reported as a Grade $4 \mathrm{AE}$, developing in more than $20 \%$ of the patients. Other CCRT regimens used in Korea, such as CCRT-VIPD [ETP, IFM, cisplatin (CDDP), and dexamethasone (DEX)] and CCRT-VIDL (ETP, IFM, DEX, and L-asp ${ }^{[37,38]}$, have common characteristics such as RT with a median of 40 Gy conducted with weekly CDDP monotherapy, and then combination chemotherapy is conducted after RT completion. VIPD is given every three weeks for three cycles after 3-5 weeks from the time of CCRT completion ${ }^{[37]}$. VIDL is similar to VIPD, but L-asp is used instead of CDDP every other day from Day 8 to Day 20, as in the SMILE regimen ${ }^{[3,42]}$. VIDL is given every three weeks for two cycles after 3-4 weeks from the time of CCRT completion ${ }^{[38]}$. Compared to the RT-2/3DeVIC regimen, these regimens include a lower dose of RT and take a longer time to complete the treatment, but both 


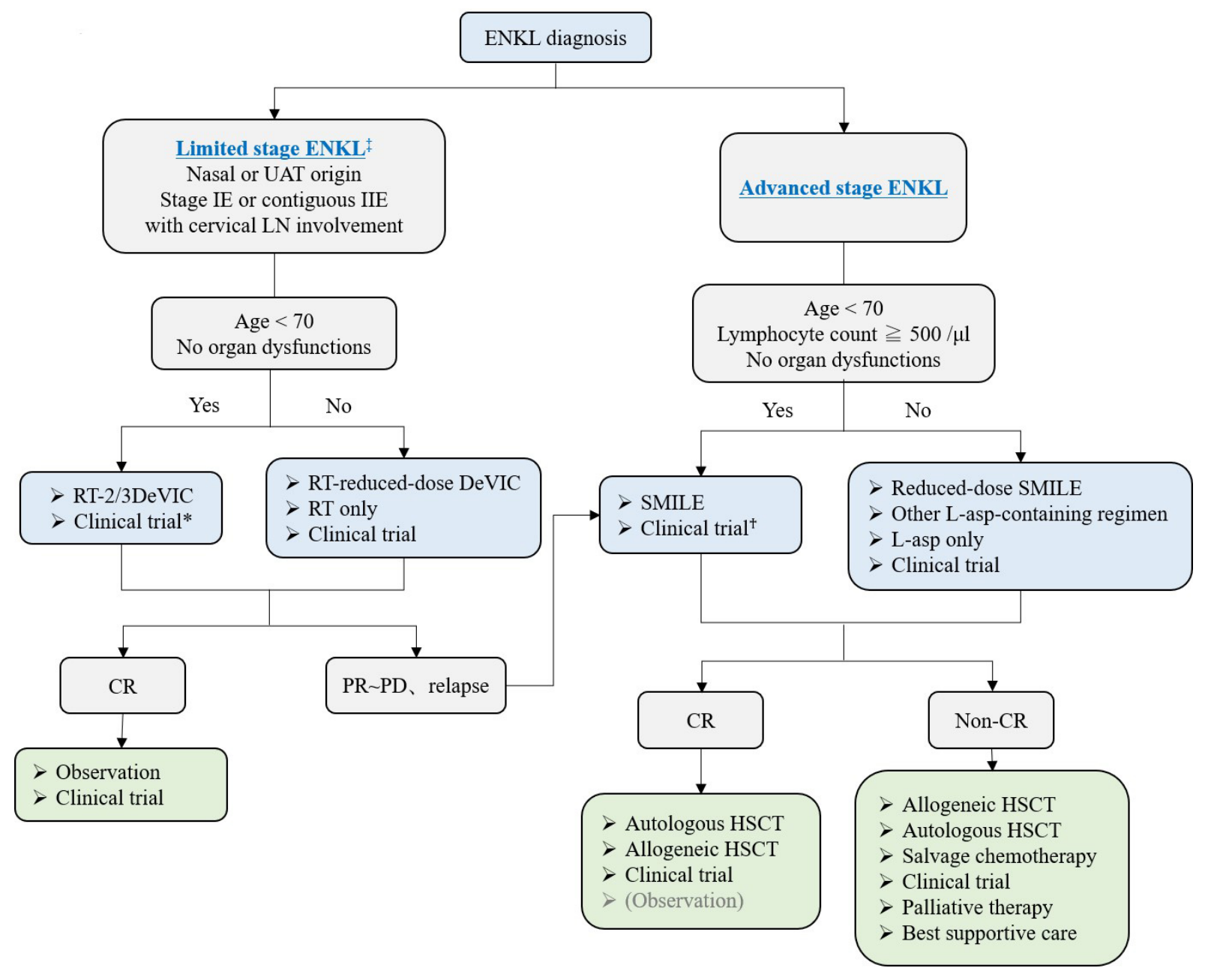

Figure 1. Treatment flowchart of ENKL patients. ${ }^{*}$ CCRT-VIPD and CCRT-VIDL regimens are included. AspaMetDex regimen is included only in relapsed or refractory ENKL patients. The definition of limited stage in this flowchart is a little different from that in the conventional Lugano/Ann Arbor classification. UAT: Upper aerodigestive tract; LN: lymph node; RT: radiotherapy; DeVIC: dexamethasone, etoposide, ifosfamide, carboplatin; CR: complete response; PR: partial response; PD: progressive disease; SMILE: steroid, methotrexate, ifosfamide, L-asparaginase, etoposide; L-asp: L-asparaginase; HSCT: hematopoietic stem cell transplantation.

results are good and comparable to those of RT-2/3DeVIC. VIDL shows less hematologic toxicity than VIPD, but there are no trials comparing these regimens. RT-2/3DeVIC is the most commonly used regimen in Japan. Recently, its durable response was reported with a five-year OS of $70 \%{ }^{[7]}$. Although there are several sequential chemoradiotherapies, no significant difference in OS has been observed compared to that with CCRT ${ }^{[43]}$, and no studies comparing an efficacy of each regimen have been reported.

\section{Advanced-stage ENKL}

For advanced stage ENKL, SMILE [steroid (DEX), methotrexate (MTX), IFM, L-asp, and ETP] chemotherapy is the most recommended regimen as a first-line therapy. Compared to that with peripheral T-cell lymphoma, because of the MDR-associated P-glycoprotein described previously herein, CHOP is much less effective for advanced-stage ENKL patients, with a five-year OS of only $8 \%-12 \%^{[5,24,44]}$. The durable efficacy of L-asp monotherapy for CHOP-resistant ENKL patients was reported in several cases ${ }^{[45,46]}$, and Lasp-containing combination chemotherapy has been developed ${ }^{[47-49]}$. The SMILE regimen, which is a nonanthracycline-containing and non-MDR-associated agent-containing combination chemotherapy, is the 
Table 1. The history of treatment development for limited and advanced stage ENKL

\begin{tabular}{|c|c|c|c|c|c|c|c|c|c|c|c|}
\hline & & Published year & Treatment & Patients & $n$ & Trial phase & ORR (\%) & CR (\%) & OS & PFS & Ref. \\
\hline & & 1998 & RT & $\mathrm{TN}$-stage $\mathrm{I} / \mathrm{II} \mathrm{NL}^{+}$ & 90 & Retrospective & NA & 74 & $48 \%-59 \%$ (2-year) & $41 \%-48 \%$ (2-year) & [24] \\
\hline \multirow[t]{12}{*}{ Limited stage } & Anthracycline-era & 2000 & RT & TN-stage I/II & 92 & Retrospective & 84 & 66 & $40 \%$ (5-year) & $38 \%$ (5-year) & [25] \\
\hline & & 2001 & $\cdot$ Frontline $\mathrm{RT} \pm \mathrm{CT}$ & TN-stage I/II & 8 & Retrospective & 100 & 100 & Median NR & NA & [32] \\
\hline & & & - Frontline $\mathrm{CT}(\mathrm{CHOP}$-like $) \pm \mathrm{RT}$ & TN-stage I/II & 12 & Retrospective & 67 & 25 & Median 35 month & NA & [32] \\
\hline & & 2001 & CHOP followed by RT 45Gy & TN-stage I/II & 17 & Retrospective & NA & 58 & $59 \%$ (3-year) & NA & [33] \\
\hline & & 2004 & $C T-> \pm R T$ & TN-stage I/II & 40 & Retrospective & NA & 72 & $29 \%$ (5-year) & $28 \%$ (5-year) & [34] \\
\hline & & 2004 & RT & TN-stage I/II & 102 & Retrospective & 85 & 72 & $42 \%$ (5-year) & $53 \%$ (5-year) & [26] \\
\hline & & 2006 & $\mathrm{RT}$ and/or CT & TN-stage IE/IIE & 105 & retrospective & 90 & 87 & $71 \%$ (5-year) & $59 \%$ (5-year) & [22] \\
\hline & & & $\rightarrow$ Frontline $\mathrm{CT}$ (CHOP-like) & & 40 & Retrospective & $60^{*}$ & $20^{*}$ & NA & NA & [22] \\
\hline & & 2007 & $\mathrm{CT}(\mathrm{CHOP})->\mathrm{RT}$ & TN-stage IE/IIE & 53 & Retrospective & NA & 49 & $76 \%$ (2-year) & $62 \%$ (2-year) & [34] \\
\hline & Non-anthracycline-era & 2009 & CCRT (RT-2/3DeVIC) & TN-stage IE/IIE & 33 & phase II & 81 & 77 & $70 \%$ (5-year) & $63 \%$ (5-year) & [36] \\
\hline & & 2009 & CCRT (RT-VIPD) & TN-stage IE/IIE & 30 & phase II & 83 & 80 & $86 \%$ (3-year) & $85 \%$ (3-year) & [37] \\
\hline & & 2014 & CCRT (RT-VIDL) & TN-stage IE/IIE & 30 & phase II & 90 & 87 & $73 \%$ (5-year) & $60 \%$ (5-year) & [38] \\
\hline \multirow[t]{8}{*}{ Advanced stage } & Anthracycline-era & 1995 & CHOP-like & TN-stage III//V NL ${ }^{+}$ & 33 & Retrospective & NA & 30 & $8 \%$ (5-year) & NA & [44] \\
\hline & & 1998 & CHOP-like & TN-stage III/IV & 9 & Retrospective & NA & NA & $12 \%$ (5-year) & NA & [24] \\
\hline & & 2003 & L-asp, Dex, VCR & CHOP-like Refractory & 18 & Retrospective & 83 & 56 & $56 \%$ (5-year) & NA & [48] \\
\hline & & 2010 & CHOP-like & TN-stage IV & 47 & Retrospective & 36 & 10 & $10 \%$ (5-year) & NA & [5] \\
\hline & Non-anthracycline-era & 2011 & SMILE & R/R or stage IV & 38 & Phase II & 79 & 45 & $50 \%$ (5-year) & NA & [42] \\
\hline & & 2011 & AspaMetDex & $R / R$ & 19 & Phase II & 78 & 61 & Median 12 month & median 12 month & [53] \\
\hline & & 2016 & P-GEMOX & $\mathrm{R} / \mathrm{R}$ or $\mathrm{TN}$-stage III/IV & 35 & Retrospective & 80 & 51 & $65 \%$ (3-year) & $39 \%$ (3-year) & [51] \\
\hline & & 2016 & Modified SMILE & TN-stage III/IV & 9 & Retrospective & 22 & NA & NA & NA & [50] \\
\hline
\end{tabular}

${ }^{\star}$ Response rate just after patients received only chemotherapy. ${ }^{+}$Nasal lymphoma includes B-, T-, or NK-cell lymphoma developing around the nasal cavity. RT: Radiotherapy; CT: chemotherapy; CCRT: concurrent chemoradiotherapy; L-asp: L-asparaginase; DEX: dexamethasone; VCR: vincristine; TN: treatment-naive; NL: nasal lymphoma; R/R: relapsed or refractory; ORR: overall response rate; CR: complete response; OS: overall survival; PFS: progression-free survival; NA: not available; ENKL: extranodal NK/T cell lymphoma, nasal type.

standard treatment for treatment-naïve advanced-stage, relapsed, or refractory ENKL ${ }^{[42]}$. In a phase 2 study, SMILE dramatically improved survival outcomes, with a one-year OS and ORR of 55\% and 79\%, respectively. Peg-asparaginase-containing regimens, including modified SMILE or P-GEMOX ${ }^{[50,51]}$, were also reported as effective treatments for advanced-stage, relapsed, or refractory ENKL, although the efficacy of these regimens was only evaluated retrospectively. The AspaMetDex (L-asp, MTX, and DEX) regimen is also listed as a suggested treatment for ENKL in the NCCN Guidelines ${ }^{[52]}$. The phase 2 study of AspaMetDex showed that the ORR was $78 \%$ and the one-year OS was $47 \%$ for relapsed or refractory ENKL patient ${ }^{[53]}$. However, the results of this regimen for treatment-naïve ENKL were disappointing with a high relapse rate because an antibody against L-asp, inhibiting its action, was observed in more than $70 \%$ of 
patients after the first cycle and eventually observed in all patients ${ }^{[54]}$. Therefore, AspaMetDex should only be used in a relapsed or refractory setting of the disease. Only a prospective clinical trial comparing the efficacy of DDGP (DEX, CDDP, gemcitabine, and peg-asparaginase) and SMILE has been reported from China, but the results cannot be evaluated owing to the poor study design ${ }^{[55]}$. The authors included a larger proportion of stage III ENKL patients than that found in the historical data (usually less than $20 \%$ of advanced-stage ENKL), and the methods and patient selection criteria described were different from the registered data on ClinicalTrials.gov (NCT01501149). Therefore, we conclude that the recommended standard treatment for advanced-stage ENKL is still the SMILE regimen ${ }^{[42]}$.

\section{Hematopoietic stem cell transplantation for ENKL}

Hematopoietic stem cell transplantation (HSCT) is recommended for relapsed or refractory limited-stage ENKL, in which a complete response (CR) is not achieved after the first-line treatment, as well as for advanced-stage ENKL. The advantage of upfront HSCT has not been established in limited-stage ENKL patients or ENKL patients with favorable risk factors who achieved first CR after induction therapy ${ }^{[56]}$. In contrast, the results of a SMILE phase 2 study suggest that the OS of patients with advanced-stage, relapsed, or refractory ENKL is better in the HSCT group than in the non-HSCT group ${ }^{[42]}$. Therefore, HSCT remains the mainstay for advanced-stage ENKL in the first CR or partial response (PR). Although autologous HSCT and allogeneic HSCT have not been compared, generally, autologous HSCT is recommended for relapsed limited-stage ENKL patients with chemo-sensitivity or advanced-stage ENKL patients who achieve first CR or PR after L-asp-containing chemotherapy, and allogeneic HSCT is recommended for the remaining patient ${ }^{[57]}$. Recently, the efficacy of four cycles of VIDL followed by up-front autologous HSCT in newly diagnosed advanced-stage ENKL patients was reported in Korea ${ }^{[58]}$. Seventeen of 24 patients prospectively included in this phase 2 study, who achieved CR or PR after four cycles of VIDL, finally proceeded with upfront autologous HSCT. The median duration of the response was 15.2 months. However, nine patients (53\%) relapsed after HSCT and four (24\%) of the nine relapsed in the central nervous system (CNS). Therefore, we have to pay attention to CNS relapse in ENKL patients who received HSCT. Further, these results suggest that advanced-stage ENKL patients, especially those with risk factors for CNS involvement, should be treated with intermediate-dose MTX-containing chemotherapy, including SMILE, and upfront HSCT $^{[59]}$. The durable efficacy of allogeneic HSCT for ENKL was reported from the Center for International Blood and Marrow Transplant Research (CIBMTR) with a three-year OS of 34\% and no relapse after two years from HSCT treatment ${ }^{[00]}$.

\section{ANKL}

A standard treatment for ANKL has not been established, and there are no prospective clinical trials for ANKL patients due to its rarity. The anthracycline-containing regimen is not effective for ANKL, with a median OS of 2-3 months ${ }^{[5]}$. SMILE therapy is recommended as a first-line treatment for ANKL, as for advanced-stage ENKL, although the reported evidence is limited. In a small-scale retrospective study, the ORR of SMILE therapy in 13 patients with ANKL was $38 \%^{[16]}$. Since ANKL progresses rapidly, most patients have B symptoms, liver dysfunction, and a poor general condition at diagnosis ${ }^{[5,12]}$. The dose of anti-cancer agents in SMILE can be reduced for such comorbid patients. Another alternative is an L-asp monotherapy followed by SMILE therapy after an improvement in organ function or the general condition. One case report showed the successful treatment with L-asp monotherapy as a first-line therapy for ANKL with severe liver dysfunction at diagnosis ${ }^{[62]}$. In that case, SMILE was supplemented after the improvement of liver function with L-asp monotherapy.

\section{Hematopoietic stem cell transplantation for ANKL}

Most ANKL patients who do not receive allogeneic HSCT, even if they achieve CR after induction chemotherapy, progress to relapse disease and eventually die $e^{[5,11,12]}$. Therefore, allogeneic HSCT is 
recommended for all ANKL patients who achieve a response and are eligible for this procedure. The effectiveness of allogeneic HSCT for ANKL was first reported in $1996^{[63]}$. Small-scale retrospective studies showing durable response and long-term survival for ANKL patients who received allogeneic HSCT have been reported since then ${ }^{[21,64,65]}$. A retrospective study of 21 ANKL patients who underwent allogeneic HSCT reported from CIBMTR showed that the two-year OS was $24 \%$ and two-year relapse rate was $59 \%$. In this study, approximately $30 \%$ of patients who achieved CR at the time of HSCT had long-term survival, whereas all who had active disease at the time of HSCT eventually died within one year due to relapse ${ }^{[6 \sigma]}$. Another retrospective study reported in Korea showed that up-front allogeneic HSCT improves survival outcomes for ANKL patients ${ }^{[67]}$. In contrast, in a small-scale retrospective study in Japan, of seven patients who underwent allogeneic HSCT and had active disease at the time of HSCT, four achieved CR after HSCT and two of these four patients remained disease-free for more than two years ${ }^{[12]}$. These results suggest that allogeneic HSCT can provide long-term survival, even in patients with active disease at the time of HSCT. Recently, the impact of allogeneic HSCT for 59 ANKL patients on survival outcomes was reported in Japan $^{[68]}$. The median OS and PFS were 3.9 and 2.6 months, respectively, which are consistent with the previous data from CIBMTR. The prognosis of patients who relapsed within one year after HSCT was poor, with a median OS of only 1.4 months. In contrast, if patients survived more than one year without relapse, the subsequent five-year OS from one year after allogeneic HSCT was good (85.2\%). The prognosis of patients who achieved CR or PR at the time of HSCT was significantly better than that of patients without a response, which was also comparable to a previous finding (five-year OS, $40.6 \% v s .16 .1 \%, P=0.046$ ). Interestingly, 15 of 24 patients with primary induction failure at the time of HSCT achieved CR after allogeneic HSCT, and the prognosis of the 15 patients (five-year OS, 32.0\%) was almost comparable with that of patients with CR or PR at HSCT $(P=0.95)$. Therefore, allogeneic HSCT should be considered for all patients with ANKL to improve survival, even for those without response at HSCT. In addition, we should try to develop novel treatments that could provide higher responses for ANKL patients, because the efficacy and response rate of the current treatments are still not satisfactory.

\section{NOVEL AGENTS AND THE POSSIBILITY OF CHEMO-FREE TREATMENTS}

The prognosis of patients with ENKL or ANKL refractory to L-asp-based regimens is extremely poor. A scheme of the associated molecular pathway and the results of clinical trials of promising novel agents for relapsed or refractory ENKL are shown in Figure 2 and Table 2, respectively. A recent study showed that, in patients with EBV-associated lymphoma, including ENKL and ANKL, PD-L1 is frequently expressed in tumor cells owing to $P D-L 1 / P D-L 2$ genetic alterations mainly in the $3^{\prime}$-untranslated regions (UTR $)^{[69,70]}$. The PD-1/PD-L1 antibody, an immune checkpoint inhibitor, is the most promising treatment for these patients. An excellent response to pembrolizumab at $100 \mathrm{mg}(2 \mathrm{mg} / \mathrm{kg})$ once every three weeks in seven patients with ENKL who relapsed or were refractory to L-asp-containing regimens was reported with the ORR of $100 \%$ and CR rate of $71 \%$, although this study had a patient selection bias ${ }^{[71]}$. Similarly, a retrospective analysis of 19 ENKL patients who were treated with pembrolizumab at $3 \mathrm{mg} / \mathrm{kg}$ once every three weeks showed that the ORR and CR rate were $47 \%$ and $37 \%$, respectively ${ }^{[72]}$. Next-generation sequencing analysis of the samples of the 19 patients before pembrolizumab initiation in this study revealed that the gene mutation occurred most frequently in the $3^{\prime}$-UTR of $P D-L 1$ (21\%), which was the only therapeutic biomarker significantly associated with a good response to pembrolizumab. All ENKL patients $(n=4)$ with a PD-L1 mutation achieved CR with pembrolizumab and sustained the response for more than 30 months. In contrast, PD-L1 expression, evaluated by immunohistochemistry, was not significantly associated with the response to pembrolizumab $^{[72]}$, which was consistent with the results of another study of seven ENKL patients treated with pembrolizumab at $100 \mathrm{mg}^{[73]}$. In this study, the ORR and CR rate were $57 \%$ and $29 \%$, respectively ${ }^{[73]}$. Regarding other immune checkpoint inhibitors, the efficacy of low-dose nivolumab, given at $40 \mathrm{mg}$ every two weeks, was also reported in a small case $\operatorname{series}^{[74]}$. In addition, sintilimab, a novel PD-1 antibody, 
Table 2. Clinical trials of single novel agent for relapsed or refractory ENKL patients

\begin{tabular}{lllllll}
\hline Agent & Target & Trial phase & $\mathbf{n}$ & ORR & CR rate & Ref. \\
\hline Pembrolizumab & PD-1 & Retrospective & 7 & 100 & 71 & {$[71]$} \\
Pembrolizumab & PD-1 & Retrospective & 19 & 47 & 37 & {$[72]$} \\
Pembrolizumab & PD-1 & Retrospective & 7 & 57 & 29 & {$[73]$} \\
Nivolumab & PD-1 & Retrospective & 3 & 67 & 67 & {$[74]$} \\
Sintilimab & PD-1 & Phase II & 28 & 68 & 14 & {$[75]$} \\
Avelumab & PD-L1 & Phase II & 21 & 38 & 24 & {$[76]$} \\
Brentuximab vedotin & CD30 & Phase II & 7 & 29 & 15 & {$[82]$} \\
Daratumumab & CD38 & Phase II & 32 & 25 & 0 & {$[83]$} \\
Chidamide & HDAC & Phase II & $79(16)$ & 19 & 6 & {$[85]$} \\
LMP1/2-specific CTL & LMP1/2 & Phase II & 6 & 67 & 67 & {$[87]$} \\
\hline
\end{tabular}

HDAC: Histone deacetylase; LMP: latent membrane protein; CTL: cytotoxic T-cells; NA: not available; ORR: overall response rate; CR: complete response; ENKL: extranodal NK/T cell lymphoma, nasal type.

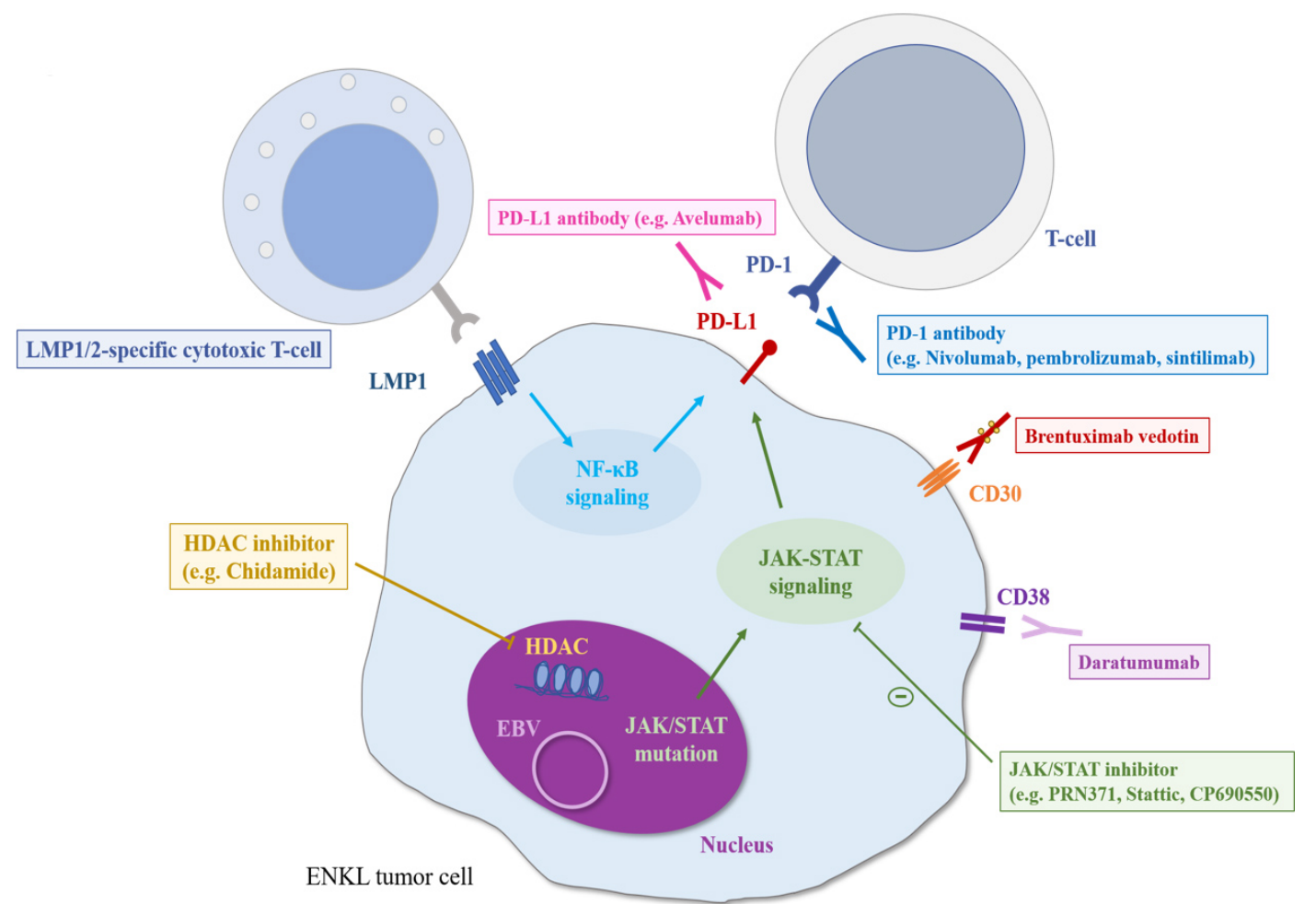

Figure 2. The scheme of molecular pathways and promising treatments for ENKL patients. LMP: Latent membrane protein; EBV: Epstein-Barr virus; HDAC: histone deacetylase.

resulted in a durable response in relapsed or refractory ENKL patients with two-year OS of $78.6 \%$ and ORR of $67.9 \%$, as well as a manageable safety profile ${ }^{[75]}$. In contrast, a slightly lower response rate to avelumab, a PD-L1 antibody, in 21 relapsed or refractory ENKL patients was reported with the ORR of only $38 \%{ }^{[76]}$. In addition, a portion of those without a response rapidly progressed. Further assessments of factors affecting the response to $\mathrm{PD}-1 / \mathrm{PD}-\mathrm{L} 1$ antibodies are needed to identify the appropriate population for those treatments. More recently, the combination of several chemotherapies with immune checkpoint inhibitors has also been evaluated and has demonstrated promising results ${ }^{[77-79]}$. In particular, a phase $\mathrm{Ib} / \mathrm{II}$ trial of the combination of sintilimab, a PD-1 antibody, and chidamide, a histone deacetylase (HDAC) inhibitor, in 36 
relapsed or refractory ENKL patients showed superior ORR and CR rates of $58 \%$ and $44 \%$, respectively ${ }^{[77]}$. Further analyses are warranted to identify the best combination therapy for ENKL patients.

Several novel targeted agents other than PD-1/PD-L1 antibodies have also been evaluated for their efficacy against ENKL. The clinical efficacy of an anti-CD30 antibody-drug conjugate (brentuximab vedotin) has been reported in two CD30-positive ENKL cases $^{[80,81]}$. A phase II study for relapsed or refractory CD30positive non-Hodgkin lymphoma, including seven ENKL patients, showed an ORR of $29 \%$ and CR rate of $15 \%{ }^{[82]}$. An anti-CD38 antibody (daratumumab) was also reported to have durable efficacy in a CD $38-$ positive ENKL case ${ }^{[83]}$. A phase II study of daratumumab monotherapy conducted for 32 relapsed or refractory ENKL patients yielded the ORR of $25 \%$ and CR rate of $0 \%$ with a median PFS of 55 days ${ }^{[84]}$. Further, the HDAC inhibitor chidamide, which was developed as an oral therapeutic in China, was evaluated in a multicenter, pivotal phase II study of 79 patients with relapsed or refractory T/NK-cell lymphoma, including ENKL patients (20\%). The ORR and CR rate of ENKL patients treated with chidamide were $19 \%$ and $6 \%$, respectively ${ }^{[85]}$. In general, ENKL is strongly associated with type II EBV latency, in which latent membrane protein 1 (LMP1) and latent membrane protein 2 (LMP2) are expressed ${ }^{[86]}$. The durable efficacy of autologous LMP1/2-specific cytotoxic T cells (CTLs) has been demonstrated for relapsed or refractory ENKL patients ${ }^{[87]}$. LMP1/2-specific CTLs were also used as a consolidative therapy for high-risk ENKL patients with a complete response after induction therapy or stem cell transplantation ${ }^{[87,88]}$. In addition, based on the findings that activation of the JAK-STAT pathway, particularly associated with STAT3 and STAT5B mutations, is observed in ENKL tumor cells ${ }^{[89-91]}$, the efficacy of a JAK3-selective inhibitor (PRN371) ${ }^{[92,93]}$ and a STAT3 inhibitor (Stattic) ${ }^{[94]}$ was reported in JAK3mutated cell lines and a xenograft model and STAT3-mutated cell lines, respectively. Moreover, a pan-JAK inhibitor (CP-690550) reduced cell viability with increased apoptosis in both JAK3-mutated and wild-type ENKL cell lines ${ }^{[93]}$. The clinical efficacies of these agents have not been well studied, and further evaluations are needed.

There are no studies evaluating the efficacy of novel targeted agents for ANKL patients. High sensitivities to a BCL2 inhibitor and a JAK2 inhibitor in vitro were reported ${ }^{[95]}$. As described previously, because the expression of PD-L1 is high in ANKL, PD-1/PD-L1 antibodies are also promising therapeutic agents for ANKL patients with failed L-asp-containing regimen treatment, and further analysis is warranted.

\section{CONCLUSION}

The prognosis of patients with disseminated NK/T cell lymphoma is markedly improving. Recent advances contribute to the improvement, but there remains room for further remedy, particularly for patients with relapsed or refractory NK/T cell lymphoma. Developing novel agents is thus warranted.

\section{DECLARATIONS}

\section{Authors' contributions}

Wrote the manuscript: Fujimoto A

Revised the manuscript: Suzuki R

\section{Availability of data and materials}

Not applicable.

\section{Financial support and sponsorship}

None. 


\section{Conflicts of Interest}

Fujimoto A declared that there are no conflicts of interest. Suzuki R received honoraria from Bristol-Myers Squibb, Novartis, Kyowa Hakko Kirin, Chugai Pharmaceuticals, Takeda, Meiji Seika Pharma, MSD, Otsuka, Celgene, Eisai Pharmaceuticals, Abbvie Inc., and Janssen outside the submitted work.

\section{Ethical approval and consent to participate}

Not applicable.

\section{Consent for publication}

Not applicable.

\section{Copyright}

(c) The Author(s) 2021.

\section{REFERENCES}

1. Campo E, Harris NL, Jaffe ES, et al. WHO classification of tumours of haematopoietic and lymphoid tissues. 4th ed. Geneva: WHO Press; 2008.

2. Lymphoma Study Group of Japanese Pathologists. The world health organization classification of malignant lymphomas in Japan: incidence of recently recognized entities. Pathol Int 2000;50:696-702. DOI

3. Bassig BA, Au WY, Mang O, et al. Subtype-specific incidence rates of lymphoid malignancies in Hong Kong compared to the United States, 2001-2010. Cancer Epidemiol 2016;42:15-23. DOI PubMed

4. Sun J, Yang Q, Lu Z, et al. Distribution of lymphoid neoplasms in China: analysis of 4,638 cases according to the World Health Organization classification. Am J Clin Pathol 2012;138:429-34. DOI PubMed

5. Suzuki R, Suzumiya J, Yamaguchi M, et al; NK-cell Tumor Study Group. Prognostic factors for mature natural killer (NK) cell neoplasms: aggressive NK cell leukemia and extranodal NK cell lymphoma, nasal type. Ann Oncol 2010;21:1032-40. DOI

6. Au WY, Weisenburger DD, Intragumtornchai T, et al; International Peripheral T-Cell Lymphoma Project. Clinical differences between nasal and extranasal natural killer/T-cell lymphoma: a study of 136 cases from the International Peripheral T-Cell Lymphoma Project. Blood 2009;113:3931-7. DOI PubMed

7. Yamaguchi M, Suzuki R, Oguchi M, et al. Treatments and outcomes of patients with extranodal natural killer/T-cell lymphoma diagnosed between 2000 and 2013: a cooperative study in Japan. J Clin Oncol 2017;35:32-9. DOI PubMed

8. Fox CP, Civallero M, Ko Y, et al. Survival outcomes of patients with extranodal natural-killer T-cell lymphoma: a prospective cohort study from the international T-cell Project. Lancet Haematol 2020;7:e284-94. DOI PubMed

9. Pongpruttipan T, Sukpanichnant S, Assanasen T, et al. Extranodal NK/T-cell lymphoma, nasal type, includes cases of natural killer cell and $\alpha \beta, \gamma \delta$, and $\alpha \beta / \gamma \delta$ T-cell origin: a comprehensive clinicopathologic and phenotypic study. Am J Surg Pathol 2012;36:481-99. DOI PubMed

10. Fernandez LA, Pope B, Lee C, Zayed E. Aggressive natural killer cell leukemia in an adult with establishment of an NK cell line. Blood 1986;67:925-30. PubMed

11. Suzuki R, Suzumiya J, Nakamura S, et al; NK-cell Tumor Study Group. Aggressive natural killer-cell leukemia revisited: large granular lymphocyte leukemia of cytotoxic NK cells. Leukemia 2004;18:763-70. DOI PubMed

12. Ishida F, Ko YH, Kim WS, et al. Aggressive natural killer cell leukemia: therapeutic potential of L-asparaginase and allogeneic hematopoietic stem cell transplantation. Cancer Sci 2012;103:1079-83. DOI PubMed PMC

13. Kimura H, Ito Y, Kawabe S, et al. EBV-associated T/NK-cell lymphoproliferative diseases in nonimmunocompromised hosts: prospective analysis of 108 cases. Blood 2012;119:673-86. DOI PubMed

14. Ko YH, Park S, Kim K, Kim SJ, Kim WS. Aggressive natural killer cell leukemia: is Epstein-Barr virus negativity an indicator of a favorable prognosis? Acta Haematol 2008;120:199-206. DOI PubMed

15. Nicolae A, Ganapathi KA, Pham TH, et al. EBV-negative aggressive NK-cell leukemia/lymphoma: clinical, pathologic, and genetic features. Am J Surg Pathol 2017;41:67-74. DOI PubMed PMC

16. Suzuki R. NK/T Cell Lymphoma: updates in therapy. Curr Hematol Malig Rep 2018;13:7-12. DOI PubMed

17. Yang Y, Wang Y, Liu X, et al. Progression-free survival at 24 months and subsequent survival of patients with extranodal NK/T-cell lymphoma: a China Lymphoma Collaborative Group (CLCG) study. Leukemia 2021;35:1671-82. DOI PubMed PMC

18. Kim SJ, Yoon DH, Jaccard A, et al. A prognostic index for natural killer cell lymphoma after non-anthracycline-based treatment: a multicentre, retrospective analysis. Lancet Oncol 2016;17:389-400. DOI PubMed

19. Lee J, Suh C, Park YH, et al. Extranodal natural killer T-cell lymphoma, nasal-type: a prognostic model from a retrospective multicenter study. J Clin Oncol 2006;24:612-8. DOI PubMed

20. Suzuki R, Suzumiya J, Oshimi K. Differences between nasal and extranasal NK/T-cell lymphoma. Blood 2009;113:6260-1; author reply 6261-2. DOI PubMed

21. Tang YT, Wang D, Luo H, et al. Aggressive NK-cell leukemia: clinical subtypes, molecular features, and treatment outcomes. Blood Cancer J 2017;7:660. DOI PubMed PMC 
22. Li YX, Yao B, Jin J, et al. Radiotherapy as primary treatment for stage IE and IIE nasal natural killer/T-cell lymphoma. J Clin Oncol 2006;24:181-9. DOI PubMed

23. Vargo JA, Patel A, Glaser SM, et al. The impact of the omission or inadequate dosing of radiotherapy in extranodal natural killer Tcell lymphoma, nasal type, in the United States. Cancer 2017;123:3176-85. DOI PubMed

24. Cheung MM, Chan JK, Lau WH, et al. Primary non-Hodgkin's lymphoma of the nose and nasopharynx: clinical features, tumor immunophenotype, and treatment outcome in 113 patients. J Clin Oncol 1998;16:70-7. DOI PubMed

25. Kim GE, Cho JH, Yang WI, et al. Angiocentric lymphoma of the head and neck: patterns of systemic failure after radiation treatment. $J$ Clin Oncol 2000;18:54-63. DOI PubMed

26. Koom WS, Chung EJ, Yang WI, et al. Angiocentric T-cell and NK/T-cell lymphomas: radiotherapeutic viewpoints. Int J Radiat Oncol Biol Phys 2004;59:1127-37. DOI PubMed

27. Yang CW, Wang CW, Hong RL, et al. Treatment outcomes of and prognostic factors for definitive radiotherapy with and without chemotherapy for Stage I/II nasal extranodal NK/T-cell lymphoma. J Radiat Res 2017;58:114-22. DOI PubMed PMC

28. Yang Y, Zhu Y, Cao JZ, et al. Risk-adapted therapy for early-stage extranodal nasal-type NK/T-cell lymphoma: analysis from a multicenter study. Blood 2015;126:1424-32; quiz 1517. DOI PubMed

29. Miller TP, Dahlberg S, Cassady JR, et al. Chemotherapy alone compared with chemotherapy plus radiotherapy for localized intermediate- and high-grade non-Hodgkin's lymphoma. N Engl J Med 1998;339:21-6. DOI PubMed

30. Egashira M, Kawamata N, Sugimoto K, et al. P-glycoprotein expression on normal and abnormally expanded natural killer cells and inhibition of P-glycoprotein function by cyclosporin A and its analogue, PSC833. Blood 1999;93:599-606. PubMed

31. Yamaguchi M, Kita K, Miwa H, et al. Frequent expression of P-glycoprotein/MDR1 by nasal T-cell lymphoma cells. Cancer 1995;76:2351-6. DOI PubMed

32. Ribrag V, Ell Hajj M, Janot F, et al. Early locoregional high-dose radiotherapy is associated with long-term disease control in localized primary angiocentric lymphoma of the nose and nasopharynx. Leukemia 2001;15:1123-6. DOI PubMed

33. Ribrag V, Ell Hajj M, Janot F, et al. Early locoregional high-dose radiotherapy is associated with long-term disease control in localized primary angiocentric lymphoma of the nose and nasopharynx. Leukemia 2001;15:1123-6. DOI PubMed

34. You JY, Chi KH, Yang MH, et al. Radiation therapy versus chemotherapy as initial treatment for localized nasal natural killer (NK)/Tcell lymphoma: a single institute survey in Taiwan. Ann Oncol 2004;15:618-25. DOI PubMed

35. Wang B, Lu JJ, Ma X, et al. Combined chemotherapy and external beam radiation for stage IE and IIE natural killer T-cell lymphoma of nasal cavity. Leuk Lymphoma 2007;48:396-402. DOI PubMed

36. Yamaguchi M, Tobinai K, Oguchi M, et al. Phase I/II study of concurrent chemoradiotherapy for localized nasal natural killer/T-cell lymphoma: Japan Clinical Oncology Group Study JCOG0211. J Clin Oncol 2009;27:5594-600. DOI PubMed

37. Kim SJ, Kim K, Kim BS, et al. Phase II trial of concurrent radiation and weekly cisplatin followed by VIPD chemotherapy in newly diagnosed, stage IE to IIE, nasal, extranodal NK/T-Cell Lymphoma: consortium for Improving Survival of Lymphoma study. J Clin Oncol 2009;27:6027-32. DOI PubMed

38. Kim SJ, Yang DH, Kim JS, et al. Concurrent chemoradiotherapy followed by L-asparaginase-containing chemotherapy, VIDL, for localized nasal extranodal NK/T cell lymphoma: CISL08-01 phase II study. Ann Hematol 2014;93:1895-901. DOI PubMed

39. Ando M, Sugimoto K, Kitoh T, et al. Selective apoptosis of natural killer-cell tumours by 1-asparaginase. Br J Haematol 2005;130:860-8. DOI PubMed

40. Chen JS, Lin KH, Lin DT, Chen RL, Jou ST, Su IJ. Longitudinal observation and outcome of nonfamilial childhood haemophagocytic syndrome receiving etoposide-containing regimens. Br J Haematol 1998;103:756-62. DOI PubMed

41. Uno M, Tsuchiyama J, Moriwaki A, et al. In vitro induction of apoptosis for nasal angiocentric natural killer cell lymphoma-derived cell line, NK-YS, by etoposide and cyclosporine A. Br J Haematol 2001;113:1009-14. DOI PubMed

42. Yamaguchi M, Kwong YL, Kim WS, et al. Phase II study of SMILE chemotherapy for newly diagnosed stage IV, relapsed, or refractory extranodal natural killer (NK)/T-cell lymphoma, nasal type: the NK-Cell Tumor Study Group study. J Clin Oncol 2011;29:4410-6. DOI PubMed

43. Kwong YL, Kim SJ, Tse E, et al. Sequential chemotherapy/radiotherapy was comparable with concurrent chemoradiotherapy for stage I/II NK/T-cell lymphoma. Ann Oncol 2018;29:256-63. DOI PubMed

44. Liang R, Todd D, Chan TK, et al. Treatment outcome and prognostic factors for primary nasal lymphoma. J Clin Oncol 1995;13:66670. DOI PubMed

45. Nagafuji K, Fujisaki T, Arima F, Ohshima K. L-asparaginase induced durable remission of relapsed nasal NK/T-cell lymphoma after autologous peripheral blood stem cell transplantation. Int J Hematol 2001;74:447-50. DOI PubMed

46. Matsumoto Y, Nomura K, Kanda-Akano Y, et al. Successful treatment with Erwinia L-asparaginase for recurrent natural killer/T cell lymphoma. Leuk Lymphoma 2003;44:879-82. DOI PubMed

47. Obama K, Tara M, Niina K. L-asparaginase-based induction therapy for advanced extranodal NK/T-cell lymphoma. Int J Hematol 2003;78:248-50. DOI PubMed

48. Yong W, Zheng W, Zhang Y, et al. L-asparaginase-based regimen in the treatment of refractory midline nasal/nasal-type T/NK-cell lymphoma. Int J Hematol 2003;78:163-7. DOI PubMed

49. Jaccard A, Petit B, Girault S, et al. L-asparaginase-based treatment of 15 western patients with extranodal NK/T-cell lymphoma and leukemia and a review of the literature. Ann Oncol 2009;20:110-6. DOI PubMed

50. Qi S, Yahalom J, Hsu M, et al. Encouraging experience in the treatment of nasal type extra-nodal NK/T-cell lymphoma in a non-Asian population. Leuk Lymphoma 2016;57:2575-83. DOI PubMed PMC

51. Wang JH, Wang L, Liu CC, et al. Efficacy of combined gemcitabine, oxaliplatin and pegaspargase (P-gemox regimen) in patients with 
newly diagnosed advanced-stage or relapsed/refractory extranodal NK/T-cell lymphoma. Oncotarget 2016;7:29092-101. DOI PubMed PMC

52. Horwitz SM, Ansell SM, Ai WZ, et al. NCCN Guidelines Insights: T-Cell Lymphomas, Version 2.2018. J Natl Compr Canc Netw 2018;16:123-35. DOI PubMed

53. Jaccard A, Gachard N, Marin B, et al; GELA and GOELAMS Intergroup. Efficacy of L-asparaginase with methotrexate and dexamethasone (AspaMetDex regimen) in patients with refractory or relapsing extranodal NK/T-cell lymphoma, a phase 2 study. Blood 2011;117:1834-9. DOI

54. Jaccard A, Suarez F, Delmer A, et al. A prospective phase II trial of an L-asparaginase containing regimen in extra nodal NK/T-cell lymphoma. Hematol Oncol 2013;31:129. DOI

55. Li X, Cui Y, Sun Z, et al. DDGP versus SMILE in newly diagnosed advanced natural killer/T-cell lymphoma: a randomized controlled, multicenter, open-label study in China. Clin Cancer Res 2016;22:5223-8. DOI PubMed

56. Yhim HY, Kim JS, Mun YC, et al; Consortium for Improving Survival of Lymphoma Study. Clinical outcomes and prognostic factors of up-front autologous stem cell transplantation in patients with extranodal natural killer/T cell lymphoma. Biol Blood Marrow Transplant 2015;21:1597-604. DOI

57. Kharfan-Dabaja MA, Kumar A, Ayala E, et al. Clinical Practice recommendations on indication and timing of hematopoietic cell transplantation in mature $\mathrm{T}$ cell and NK/T cell lymphomas: an International Collaborative Effort on Behalf of The Guidelines Committee of The American Society for blood and marrow transplantation. Biol Blood Marrow Transplant 2017;23:1826-38. DOI PubMed

58. Song GY, Yoon DH, Suh C, et al. Open-label, single arm, multicenter phase II study of VIDL induction chemotherapy followed by upfront autologous stem cell transplantation in patients with advanced stage extranodal NK/T-cell lymphoma. Bone Marrow Transplant 2021;56:1205-8. DOI PubMed PMC

59. Kim H, Jeong H, Yamaguchi M, et al. Prediction and prevention of central nervous system relapse in patients with extranodal natural killer/T-cell lymphoma. Blood 2020;136:2548-56. DOI PubMed

60. Kanate AS, DiGilio A, Ahn KW, et al. Allogeneic haematopoietic cell transplantation for extranodal natural killer/T-cell lymphoma, nasal type: a CIBMTR analysis. Br J Haematol 2018;182:916-20. DOI PubMed PMC

61. Jung KS, Cho SH, Kim SJ, Ko YH, Kang ES, Kim WS. L-asparaginase-based regimens followed by allogeneic hematopoietic stem cell transplantation improve outcomes in aggressive natural killer cell leukemia. J Hematol Oncol 2016;9:41. DOI PubMed PMC

62. Takahashi H, Sakai R, Hattori Y, et al. Successful disease control with L-asparaginase monotherapy for aggressive natural killer cell leukemia with severe hepatic failure. Leuk Lymphoma 2013;54:662-4. DOI PubMed

63. Teshima T, Miyaji R, Fukuda M, Ohshima K. Bone-marrow transplantation for Epstein-Barr-virus-associated natural killer cell-large granular lymphocyte leukaemia. Lancet 1996;347:1124. DOI PubMed

64. Murashige N, Kami M, Kishi Y, et al. Allogeneic haematopoietic stem cell transplantation as a promising treatment for natural killercell neoplasms. Br J Haematol 2005;130:561-7. DOI PubMed

65. Ito T, Makishima H, Nakazawa H, et al. Promising approach for aggressive NK cell leukaemia with allogeneic haematopoietic cell transplantation. Eur J Haematol 2008;81:107-11. DOI PubMed

66. Hamadani M, Kanate AS, DiGilio A, et al. Allogeneic hematopoietic cell transplantation for aggressive NK cell leukemia. A center for international blood and marrow transplant research analysis. Biol Blood Marrow Transplant 2017;23:853-6. DOI PubMed PMC

67. Jeong SH, Song HN, Park JS, et al. Allogeneic stem cell transplantation for patients with natural killer/T cell lymphoid malignancy: a multicenter analysis comparing upfront and salvage transplantation. Biol Blood Marrow Transplant 2018;24:2471-8. DOI PubMed

68. Fujimoto A, Ishida F, Izutsu K, et al. Allogeneic stem cell transplantation for patients with aggressive NK-cell leukemia. Bone Marrow Transplant 2021;56:347-56. DOI PubMed

69. Chen BJ, Chapuy B, Ouyang J, et al. PD-L1 expression is characteristic of a subset of aggressive B-cell lymphomas and virusassociated malignancies. Clin Cancer Res 2013;19:3462-73. DOI PubMed PMC

70. Kataoka K, Miyoshi H, Sakata S, et al. Frequent structural variations involving programmed death ligands in Epstein-Barr virusassociated lymphomas. Leukemia 2019;33:1687-99. DOI PubMed PMC

71. Kwong YL, Chan TSY, Tan D, et al. PD1 blockade with pembrolizumab is highly effective in relapsed or refractory NK/T-cell lymphoma failing 1-asparaginase. Blood 2017;129:2437-42. DOI PubMed

72. Lim JQ, Huang D, Tang T, et al. Whole-genome sequencing identifies responders to Pembrolizumab in relapse/refractory naturalkiller/T cell lymphoma. Leukemia 2020;34:3413-9. DOI PubMed PMC

73. Li X, Cheng Y, Zhang M, et al. Activity of pembrolizumab in relapsed/refractory NK/T-cell lymphoma. J Hematol Oncol 2018;11:15. DOI PubMed PMC

74. Chan TSY, Li J, Loong F, Khong PL, Tse E, Kwong YL. PD1 blockade with low-dose nivolumab in NK/T cell lymphoma failing Lasparaginase: efficacy and safety. Ann Hematol 2018;97:193-6. DOI PubMed

75. Li J, Tao R, Fan L, et al. Sintilimab for relapsed/refractory (r/r) extranodal NK/T cell lymphoma (ENKTL): extended follow-up on the multicenter, single-arm phase II trail (ORIENT-4). J Clin Oncol 2020;38:8050. DOI

76. Kim SJ, Lim JQ, Laurensia Y, et al. Avelumab for the treatment of relapsed or refractory extranodal NK/T-cell lymphoma: an openlabel phase 2 study. Blood 2020;136:2754-63. DOI PubMed

77. Gao Y, Huang H, Wang X, et al. Anti-PD-1 antibody (Sintilimab) plus histone deacetylase inhibitor (Chidamide) for the treatment of refractory or relapsed extranodal natural killer/T cell lymphoma, nasal type (r/r-ENKTL): preliminary results from a prospective, multicenter, single-arm, phase Ib/II trial (SCENT). Blood 2020;136:39-40. DOI

78. Cai J, Liu P, Huang H, et al. Combination of anti-PD-1 antibody with P-GEMOX as a potentially effective immunochemotherapy for 
advanced natural killer/T cell lymphoma. Signal Transduct Target Ther 2020;5:289. DOI PubMed PMC

79. Du L, Zhang L, Li L, et al. Effective treatment with PD-1 antibody, chidamide, etoposide, and thalidomide (PCET) for relapsed/refractory natural killer/T-cell lymphoma: a report of three cases. Onco Targets Ther 2020;13:7189-97. DOI PubMed PMC

80. Kim HK, Moon SM, Moon JH, Park JE, Byeon S, Kim WS. Complete remission in CD30-positive refractory extranodal NK/T-cell lymphoma with brentuximab vedotin. Blood Res 2015;50:254-6. DOI PubMed PMC

81. Poon LM, Kwong YL. Complete remission of refractory disseminated NK/T cell lymphoma with brentuximab vedotin and bendamustine. Ann Hematol 2016;95:847-9. DOI PubMed

82. Kim SJ, Yoon DH, Kim JS, et al. Efficacy of brentuximab vedotin in relapsed or refractory high-CD30-expressing non-Hodgkin lymphomas: results of a multicenter, open-labeled phase II trial. Cancer Res Treat 2020;52:374-87. DOI PubMed PMC

83. Hari P, Raj RV, Olteanu H. Targeting CD38 in refractory extranodal natural killer cell-T-cell lymphoma. $N$ Engl J Med 2016;375:1501-2. DOI PubMed

84. Huang H-q, Kim W-S, Yao M, et al. Daratumumab monotherapy for patients with relapsed or refractory (R/R) natural killer/T-cell lymphoma (NKTCL), nasal type: updated results from an open-label, single-arm, multicenter phase 2 study. Blood 2019;134:1568. DOI

85. Shi Y, Dong M, Hong X, et al. Results from a multicenter, open-label, pivotal phase II study of chidamide in relapsed or refractory peripheral T-cell lymphoma. Ann Oncol 2015;26:1766-71. DOI

86. Fujimoto A, Suzuki R. Epstein-Barr virus-associated post-transplant lymphoproliferative disorders after hematopoietic stem cell transplantation: pathogenesis, risk factors and clinical outcomes. Cancers (Basel) 2020;12:328. DOI PubMed PMC

87. Bollard CM, Gottschalk S, Torrano V, et al. Sustained complete responses in patients with lymphoma receiving autologous cytotoxic T lymphocytes targeting Epstein-Barr virus latent membrane proteins. J Clin Oncol 2014;32:798-808. DOI PubMed PMC

88. Cho SG, Kim N, Sohn HJ, et al. Long-term outcome of extranodal NK/T cell lymphoma patients treated with postremission therapy using EBV LMP1 and LMP2a-specific CTLs. Mol Ther 2015;23:1401-9. DOI PubMed PMC

89. Huang Y, de Reyniès A, de Leval L, et al. Gene expression profiling identifies emerging oncogenic pathways operating in extranodal NK/T-cell lymphoma, nasal type. Blood 2010;115:1226-37. DOI PubMed PMC

90. Lee S, Park HY, Kang SY, et al. Genetic alterations of JAK/STAT cascade and histone modification in extranodal NK/T-cell lymphoma nasal type. Oncotarget 2015;6:17764-76. DOI PubMed PMC

91. Küçük C, Jiang B, Hu X, et al. Activating mutations of STAT5B and STAT3 in lymphomas derived from $\gamma \delta$-T or NK cells. Nat Commun 2015;6:6025. DOI

92. Nairismägi ML, Gerritsen ME, Li ZM, et al. Oncogenic activation of JAK3-STAT signaling confers clinical sensitivity to PRN371, a novel selective and potent JAK3 inhibitor, in natural killer/T-cell lymphoma. Leukemia 2018;32:1147-56. DOI PubMed PMC

93. Koo GC, Tan SY, Tang T, et al. Janus kinase 3-activating mutations identified in natural killer/T-cell lymphoma. Cancer Discov 2012;2:591-7. DOI PubMed

94. Sim SH, Kim S, Kim TM, et al. Novel JAK3-activating mutations in extranodal NK/T-cell lymphoma, nasal type. Am J Pathol 2017;187:980-6. DOI PubMed

95. Dufva O, Kankainen M, Kelkka T, et al. Aggressive natural killer-cell leukemia mutational landscape and drug profiling highlight JAK-STAT signaling as therapeutic target. Nat Commun 2018;9:1567. DOI PubMed PMC 